1980-01-01

\title{
The Dufour effect. III. Direct experimental determination of the heat of transport of carbon tetrachloride-cyclohexane liquid mixtures
}

\author{
Richard L. Rowley \\ rowley@byu.edu \\ Frederick H. Horne
}

Follow this and additional works at: https://scholarsarchive.byu.edu/facpub

Part of the Chemical Engineering Commons

\section{Original Publication Citation}

R.L. Rowley and F.H. Horne, "The Dufour Effect. III. Direct Experimental Determination of Heats of Transport for the Carbon Tetrachloride Cyclohexane System", J. Chem. Phys. 72, 131 (198)

\section{BYU ScholarsArchive Citation}

Rowley, Richard L. and Horne, Frederick H., "The Dufour effect. III. Direct experimental determination of the heat of transport of carbon tetrachloride-cyclohexane liquid mixtures" (1980). Faculty Publications. 768.

https://scholarsarchive.byu.edu/facpub/768

This Peer-Reviewed Article is brought to you for free and open access by BYU ScholarsArchive. It has been accepted for inclusion in Faculty Publications by an authorized administrator of BYU ScholarsArchive. For more information, please contact ellen_amatangelo@byu.edu. 


\title{
The Dufour effect. III. Direct experimental determination of the heat of transport of carbon tetrachloride-cyclohexane liquid mixtures
}

\author{
Richard L. Rowley a) and Frederick H. Horne \\ Department of Chemistry, Michigan State University, East Lansing, Michigan 48824 \\ (Received 6 July 1979; accepted 5 September 1979) \\ The heat of transport of carbon tetrachloride-cyclohexane liquid mixtures has been determined directly \\ by Dufour effect experiments. The technique employs a withdrawable "liquid gate" to create a \\ nonturbulent, sharp diffusional interface. The partial differential equations governing the barycentric \\ velocity, composition, and temperature distributions are solved with a Crank-Nicholson implicit \\ numerical scheme. This allows inclusion of the composition and temperature dependence of the \\ thermodynamic and transport parameters. For mean mole fraction of carbon tetrachloride $x_{1}$ between \\ 0.34 and 0.55 and for mean temperature $T$ between 295.13 and $296.43^{\circ} \mathrm{K}$, the best least squares fit of \\ the data for the heat of transport $\bar{Q}^{*}{ }_{1}$ in $\mathrm{kJ} \mathrm{mol}^{-1}$ is $\tilde{Q}^{*}{ }_{1}=5.82+2.32\left(x_{1}-1 / 2\right)+0.225(T-295.82)$, \\ with a calculated standard error of 0.087 . Analysis of the same experiments indicates that the \\ composition dependence of the thermal conductivity $\kappa$ of the mixture in the specified composition and \\ temperature ranges is best given (in $\left.\mathrm{J} \mathrm{m}^{-1} s^{-1} K-1\right)$ by $\left(\partial \kappa / \partial w_{1}\right)_{T, p}=0.0252\left(w_{2}^{1 / 2}-0.032\right)$, where $w_{i}$ is \\ mass fraction.
}

\section{INTRODUCTION}

The linear relations between fluxes and independent forces for a binary, isotropic, nonelectrolyte system $\operatorname{are}^{1}$

$$
\begin{aligned}
& -\jmath_{1}=\Omega_{10} \nabla \ln T+\Omega_{11} \nabla_{T}\left(\bar{\mu}_{1}-\bar{\mu}_{2}\right), \\
& -q=\Omega_{00} \nabla \ln T+\Omega_{01} \nabla_{T}\left(\bar{\mu}_{1}-\bar{\mu}_{2}\right),
\end{aligned}
$$

where $q$ is the heat flux, $\jmath_{1}$ is the diffusion flux, $\bar{\mu}_{i}$ is the specific chemical potential of component $i, \nabla_{T} \bar{\mu}_{i}$ $\equiv \nabla \bar{\mu}_{i}+\bar{S}_{i} \nabla T\left(\bar{S}_{i}\right.$ is partial specific entropy), $T$ is temperature, and the $\Omega_{i j}$ are Onsager coefficients.

Thermal diffusion (Soret effect) and the diffusion thermoeffect (Dufour effect) are related to the coefficients $\Omega_{10}$ and $\Omega_{01}$, respectively. Equation (1.1) shows that the rmal diffusion is the diffusional flux induced by a gradient of temperature in a system of initially uniforn chemical potential. The Dufour effect is the heat flux produced in an initially isothermal mixture by the chemical potential gradient shown in Eq. (1.2). To emphasize this inverse phenomenology, the Dufour effect is often called the diffusion thermoeffect. An example of Onsager reciprocity is the equality of $\Omega_{10}$ and $\Omega_{01}$. Though originally based on microscopic timereversal concepts, ${ }^{2}$ we recently reported ${ }^{3}$ the first experimental verification that $\Omega_{10}=\Omega_{01}$ in liquids.

Traditionally, heats of transport of liquid mixtures have been obtained only from thermal diffusion experiments on the assumption of Onsager reciprocity. We report here a method for the direct determination of heats of transport by diffusion thermoeffect experiments, and we report values obtained for liquid mixtures of carbon tetrachloride and cyclohexane of various compositions. Nonlinear least-squares parameter estimation was used to obtain heat of transport data from measured temperatures and numerical solutions to the

\footnotetext{
a) Present address: Department of Chemical Engineering, William Marsh Rice University, Houston, Texas 77001.
}

partial differential equations which mathematically describe the experiment.

Although the Dufour effect in gases has been studied under a variety of conditions, ${ }^{4,5}$ the effect in liquids was long thought to be too small to study quantitatively because of the relatively larger thermal conductivity and heat of mixing effects in liquid systems. The only previously reported attempts to observe the Dufour effect in liquids were by Rastogi et al. ${ }^{6}$ As previously discussed, ${ }^{3,7}$ their experimental geometry was not amenable to theoretical analysis. In fact, most of the desired effect was eliminated by the cell design.

Ingle and Horne ${ }^{7}$ solved the coupled, nonlinear, nonhomogeneous partial differential equations which describe the Dufour effect by means of an analytical double perturbation scheme. Their prediction that the Dufour effect should be measurable in liquids arose from its antisymmetric behavior about the interface in contrast to the symmetric effect of the heat of mixing term. Their double perturbation scheme deferred the nonlinear contributions to higher perturbation orders where they became a nonhomogeneous contribution. The composition and temperature dependence of the various parameters were thus completely included, in principle. The number of terms required and the rapidly increasing complexity of successively higher order terms limit the practical application of the Ingle and Horne technique to those liquid systems with properties only slightly temperature and composition dependent.

Due to the relatively large composition and temperature dependences of the thermodynamic and transport parameters for the carbon tetrachloride-cyclohexane system, a numerical solution was used to obtain the heats of transport reported in this paper. The solutions obtained by means of a Crank-Nicholson implicit finite differencing scheme show that the composition dependences of the thermal conductivity and the heat of transport are important in the determination of the heat of transport. Asymmetries in temperature-time profiles 
observed both by us and by investigators of gaseous system $s^{5}$ are due chiefly to nonconstancy of parameters with respect to composition.

\section{THEORETICAL}

When an interface between two phases of an isothermal binary mixture is suddenly formed, a diffusion flux $j_{1}$ and a heat flux $q$ begin because of the existence of a chemical potential gradient. A measure of the heat transported by diffusion is

$$
\bar{Q}_{1}^{*}=\left(q / j_{1}\right)_{\Delta T=0},
$$

where $\bar{Q}_{1}^{*}$ is the specific heat of transport. Since direct measurement of fluxes is difficult, we derive from hydrodynamics and nonequilibrium thermodynamics partial differential equations which relate $\bar{Q}_{1}^{*}$ to more readily measurable temperature and composition distributions.

The hydrodynamic conservation equations may be written in one dimension for an experimental cell in which the height to diameter ratio is small and the lateral walls of which are adiabatic. The conservation-ofmass equations are then ${ }^{1,7}$

$$
\begin{aligned}
& (\partial \rho / \partial t)=-(\partial \rho v / \partial z), \\
& \rho\left(\partial v w_{1} / \partial t\right)+\left(\partial j_{1} / \partial z\right)+\rho v\left(\partial w_{1} / \partial z\right)=0,
\end{aligned}
$$

where $\rho$ is density, $v$ is barycentric velocity, $w_{1}$ is mass fraction of component $1, t$ is time, $z$ is the vertical coordinate with $0 \leq(z / a) \leq 1$, and $a$ is cell height.

The equation for energy transport, ${ }^{1,7}$ provided the mixture is isotropic, nonreacting, and fieldfree, is, in terms of measurable properties,

$$
\begin{gathered}
\rho \bar{C}_{p}[(\partial T / \partial t)+v(\partial T / \partial z)]-T \alpha[(\partial p / \partial t)+v(\partial p / \partial z)] \\
=\phi_{1}-(\partial q / \partial z)-j_{1}\left[\partial\left(\bar{H}_{1}-\bar{H}_{2}\right) / \partial z\right],
\end{gathered}
$$

where $\bar{C}_{p}$ is specific heat capacity, $T$ is temperature, $\alpha$ is thermal expansivity, $p$ is pressure, $\phi_{1}$ is the entropy source term for bulk flow, and $\bar{H}_{i}$ is partial specific enthalpy of component $i$.

The flux equations, Eqs. (1.1) and (1.2), can be rewritten in terms of composition variables and transport parameters by transformation of chemical potential derivatives to mass fraction and pressure gradients, ${ }^{7}$

$-j_{1}=\rho D\left(\partial w_{1} / \partial z\right)-\rho D \alpha_{1} w_{1} w_{2}(\partial \ln T / \partial z)+\rho D S_{1} w_{1} w_{2}(\partial p / \partial z)$,

$-q={ }_{k}(\partial T / \partial z)+\rho D \bar{Q}_{1}^{*}\left(\partial w_{1} / \partial z\right)+\rho D \bar{Q}_{1}^{*} S_{1} w_{1} w_{2}(\partial p / \partial z)$,

where $D$ is the mutual diffusion coefficient, $\alpha_{1}$ is the thermal diffusion factor, $S_{1}$ is the sedimentation ceofficient of component 1 , and $\kappa$ is the thermal conductivity. In Eqs. (2.5) and (2.6), the Onsager coefficients have been replaced by equivalent ${ }^{1}$ expressions in terms of transport parameters:

$$
\begin{array}{ll}
\Omega_{00}=\kappa T, & \Omega_{01}=\rho D \bar{Q}_{1}^{*} w_{1}\left(\partial \bar{\mu}_{1} / \partial w_{1}\right)^{-1}, \\
\Omega_{10}=-\rho w_{1} w_{2} \alpha_{1} D, & \Omega_{11}=\rho w_{2} D\left(\partial \bar{\mu}_{1} / \partial w_{1}\right)^{-1} .
\end{array}
$$

In Eq. (2.4), the pressure terms, the thermal diffusion term, and the bulk flow entropy source term $\phi_{1}$ are demonstrably negligible for normal liquids. ${ }^{7}$ For most nonelectrolyte liquid mixtures, the thermodynamic and transport parameters are less composition dependent when expressed as molar rather than as specific quantities. Transformation of Eqs. (2.2)-(2.4) to mole fractions and molar quantities with the aid of Eqs. (2.5), (2.6), and the above simplifications results in

$$
\begin{aligned}
(\partial v / \partial z) & \left.=\left(M_{2} \tilde{V}_{1}-M_{1} \tilde{V}_{2}\right)\left\{\partial[D / \tilde{V} \tilde{M})\left(\partial x_{1} / \partial z\right)\right] / \partial z\right\} \\
\left(\partial x_{1} / \partial t\right) & \left.=\tilde{V} \tilde{M}\left\{\partial[D / \tilde{V} \tilde{M})\left(\partial x_{1} / \partial z\right)\right] / \partial z\right\}-v\left(\partial x_{1} / \partial z\right) . \\
(\partial T / \partial t) & =\left(\tilde{V} / \tilde{C}_{p}\right)\{\partial[\kappa(\partial T / \partial z)] / \partial z\} \\
& +\left(M_{2} \tilde{V} / \tilde{C}_{p}\right)\left\{\partial\left[\left(D Q_{1}^{*} / \tilde{V} \tilde{M}\right)\left(\partial x_{1} / \partial z\right)\right] / \partial z\right\} \\
& +\left(D / \tilde{C}_{p}\right)\left(\partial^{2} \tilde{H}^{E} / \partial x_{1}^{2}\right)_{T, P}\left(\partial x_{1} / \partial z\right)^{2}-v(\partial T / \partial z),
\end{aligned}
$$

where $\tilde{M}=x_{1} M_{1}+x_{2} M_{2}$ is the mean molecular weight, $\tilde{V}$ is the molar volume, $\tilde{C}_{p}$ is the molar constant pressure heat capacity, $\bar{Q}_{1}^{*}$ is the molar heat of transport, and $\tilde{H}^{E}$ is the molar excess enthalpy. Equations (2.8)-(2.10) are the mole fraction-molar property versions of the mass fraction-specific property Eqs. (2.9)-(2.11) of DE.I. ${ }^{7}$ The excess molar enthalpy $\tilde{H}^{E}$ is related to the difference in partial specific enthalpies used in DE. I by $^{8}$

$\left[\partial\left(\bar{H}_{1}-\bar{H}_{2}\right) / \partial z\right]=\left(\tilde{M} / M_{1} M_{2}\right)\left(\partial^{2} \tilde{H}^{E} / \partial x_{1}^{2}\right)_{T, p}\left(\partial x_{1} / \partial z\right)$.

[Equation (B3) of DE. I is incorrect. The correct equation is

$$
\left(\partial / \partial w_{1}\right)=\left(\bar{M}^{2} / M_{1} M_{2}\right)\left(\partial / \partial x_{1}\right) .
$$

This leads to the present Eq. (2.11) rather than to the incorrect Eq. (B6) of DE.I.]

For carbon tetrachloride-cyclohexane mixtures, the excess volume of mixing is very small (see Table I). Thus, $\tilde{V}_{i}=\tilde{V}_{i}^{0}$, and the integration of Eq. (2.8) yields

$$
v=\left(M_{2} \tilde{V}_{1}^{0}-M_{1} \tilde{V}_{2}^{0}\right)(D / \tilde{V} \bar{M})\left(\partial x_{1} / \partial z\right),
$$

where we have also used the boundary conditions

$$
\begin{aligned}
& v(0, t)=0=v(a, t), \\
& \left(\partial x_{1} / \partial z\right)_{0, t}=0=\left(\partial x_{1} / \partial z\right)_{a_{1} t} .
\end{aligned}
$$

Substitution of Eq. (2.12) into Eq. (2.9) and rearrangement yields

$$
\begin{aligned}
\left(\partial x_{1} / \partial t\right)= & D\left(\partial^{2} x_{1} / \partial z^{2}\right) \\
& +\left[\left(\partial D / \partial x_{1}\right)_{T, p}-2(D / \tilde{V})\left(\partial \tilde{V} / \partial x_{1}\right)_{T, P}\right]\left(\partial x_{1} / \partial z\right)^{2} .
\end{aligned}
$$

In the experiments reported here, the composition and temperature dependences of $D$ and $\bar{V}$ in Eq. (2.15) do not contribute measurably to the observed temperature difference produced by the diffusion thermoeffect. We have verified this statement by numerical computations in which the heat of transport was determined (from the experimental temperature differences) both with and without the composition and temperature dependences of $D$ and $\tilde{V}$. No detectable effect was found. Thus, it suffices to use

$$
\left(\partial x_{1} / \partial t\right)=D_{0}\left(\partial^{2} x_{1} / \partial z^{2}\right),
$$

instead of Eq. (2.15) to describe the composition distribution in space and time. In Eq. (2.16) and elsewhere in this paper, the subscript 0 denotes the value of the 
TABLE I. Literature values for properties of the carbon tetrachloride-cyclohexane system at 1 atm. The properties are expressed in the general form

$$
L=L_{0}\left[1+L_{x}\left(x_{1}-0.5\right)+L_{T}(T-298.15)+L_{x T}\left(x_{1}-0.5\right)(T-298.15)+\frac{1}{2} L_{x x}\left(x_{1}=0.5\right)^{2}\right] .
$$

A. Properties of the pure components and of equimolar mixtures at $298.15^{\circ} \mathrm{K}$

\begin{tabular}{|c|c|c|c|c|c|}
\hline Property & $L$ & $L_{1}^{0}$ & $L_{2}^{0}$ & $L_{0}$ & Units \\
\hline Molecular weight ${ }^{\mathrm{a}}$ & $\tilde{M}$ & 0.15384 & 0.08418 & 0.11901 & $\mathrm{~kg} \mathrm{~mol}-1$ \\
\hline Diffusivity $^{\mathrm{b}}$ & $D$ & 1. $295 \times 10^{-9}$ & 1. $482 \times 10^{-9}$ & $1.388 \times 10^{-9}$ & $\mathrm{~m}^{2} \mathrm{~s}^{-1}$ \\
\hline Heat of Mixing ${ }^{c}$ & $\left(\partial^{2} \tilde{H}^{E} / \partial x_{1}^{2}\right)_{T, P}$ & $\cdots$ & $\cdots$ & -1.535 & $\mathrm{~kJ} \mathrm{~mol}^{-1}$ \\
\hline Heat Capacity ${ }^{\mathrm{d}}$ & $\tilde{C}$ & 131.82 & 156.76 & 144.14 & $\mathrm{~J} \mathrm{~mol}^{-1} \mathrm{~K}^{-1}$ \\
\hline Molar Volume $e^{e}$ & $\tilde{V}$ & $0.9708 \times 10^{-4}$ & 1. $0867 \times 10^{-4}$ & $1.0287 \times 10^{-4}$ & $\mathrm{~m}^{3} \mathrm{~mol}^{-1}$ \\
\hline Thermal Conductivity & $\kappa$ & 0.103 & 0.119 & $\cdots$ & $\mathrm{J} \mathrm{m}^{-1} \mathrm{~s}^{-1} \mathrm{~K}^{-1}$ \\
\hline
\end{tabular}

B. Composition and temperature coefficients

\begin{tabular}{llcccc} 
Property & $L$ & \multicolumn{1}{c}{$L_{x}$} & \multicolumn{1}{c}{$L_{T} /{ }^{\circ} \mathrm{K}$} & $L_{x T} \cdot{ }^{\circ} \mathrm{K}$ & $L_{\mathbf{x x}}$ \\
\hline Molecular Weight ${ }^{\mathrm{a}}$ & $\tilde{M}$ & 0.585 & $\cdots$ & $\cdots$ & $\cdots$ \\
Diffusivity $^{\mathrm{b}}$ & $D$ & -0.135 & 0.0185 & 0.0017 & $\ldots$ \\
Heat of Mixing $^{\mathrm{c}}$ & $\left({ }^{2} \tilde{H}^{E} / \partial_{x}^{2}\right)_{T, P}$ & -0.675 & -0.0032 & -0.0012 & 0.857 \\
Heat capacity $^{\mathrm{d}}$ & $\tilde{C}_{p}$ & -0.173 & 0.0015 & -0.0025 & 0.008 \\
Molar Volume $^{\mathrm{e}}$ & $\tilde{V}$ & -0.113 & 0.0012 & -0.0001 & $\cdots$ \\
Thermal Conductivity $^{\mathrm{f}}$ & $\kappa$ & $\ldots$ & -0.0019 & $\ldots$ & $\ldots$ \\
\hline
\end{tabular}

${ }^{\text {aReference } 11 .}$

beference 12

${ }^{c}$ References 13 and 14.

${ }^{\mathrm{d}}$ Reference 14.

References 14 and 15 .

${ }^{1}$ Reference 16.

coefficient at $x_{1}=0.5$ and $T=298.15^{\circ} \mathrm{K}$. Explicit formulas for calculating directly the effect of the composition and temperature dependences of $D$ and $\tilde{V}$ on the experimentally observed temperature difference may be found in DE. I. ${ }^{7}$ As emphasized there, these dependencies contribute very little to the temperature difference because they contribute terms which are mainly symmetric about the center of the cell.

The solution of Eq. (2.16), subject to the experimetal boundary conditions, is ${ }^{7}$

$$
\begin{aligned}
x_{1}= & \left\langle x_{1}\right\rangle+\left(\Delta x_{1}\right)(2 / \pi) \sum_{l=0}^{\infty}(-1)^{l}(2 l+1)^{-1} \\
& \times\left\{\exp \left[-(2 l+1)^{2}(t / \theta)\right]\right\} \cos [(2 l+1) \pi z / a],
\end{aligned}
$$

where $\left\langle x_{1}\right\rangle$ is the initial arithmetic mean $x_{1}, \Delta x_{1}$ is the initial difference in $x_{1}$ between the top half of the cell and the bottom half, and $\theta \equiv a^{2} / \pi^{2} D_{0}$, where $a$ is the cell height.

The general numerical scheme for solving parabolic partial differential equations, as outlined in the next section, can be used with either Eq. (2.15), or Eq. (2.16). A typical solution of the composition equation is illustrated in Fig. 1. Comparison of the numerical solution of Eq. (2.16) to its analytical solution, Eq. (2.17), provided a check on the numerical technique. The barycentric velocity distribution from Eqs. (2.12) and (2.17) is shown in Fig. 2. The initial conditions are given under Fig. 1.

The temperature equation Eq. (2.10) can be simplified by introducing into it Eqs. (2.12) and (2.17). The resulting equation is still quite complicated, and its similarly complicated solution was reported in $\mathrm{DE} . \mathrm{I}^{7}$ in Eqs. (5.16), (7.2), and (A2). For the results reported in this paper, we have solved both the temperature and composition equations numerically for the particular system carbon tetrachloride-cyclohexane.

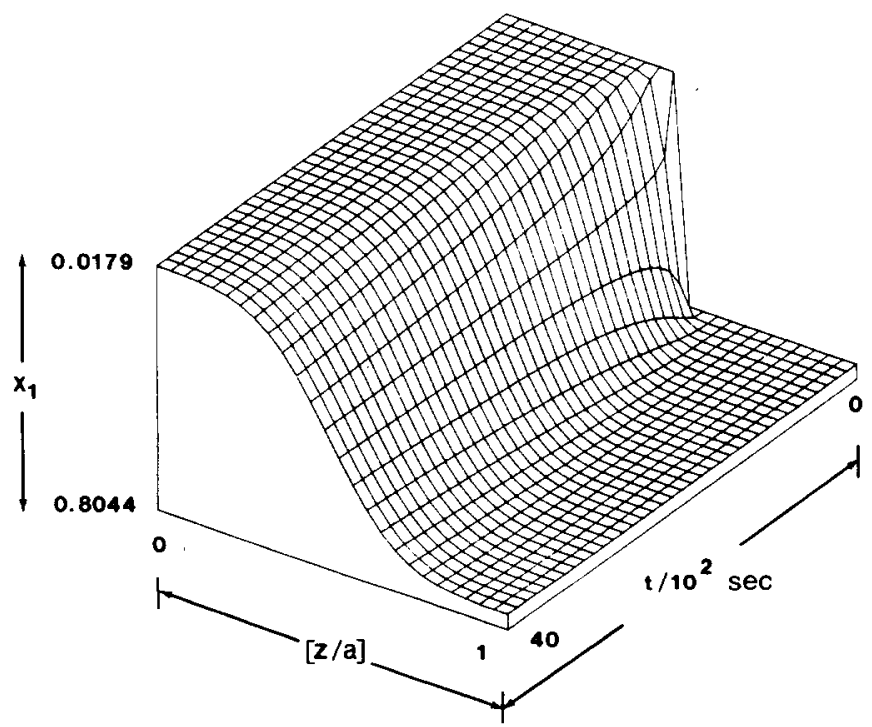

FIG. 1. Compos tion surface for the carbon tetrachloridecyclohexane system for Run I. Component 1 is $\mathrm{CCl}_{4}$. Initial conditions: $x_{1}^{U}=0.0179, x_{1}^{F}=0.8044, T=296.16^{\circ} \mathrm{K}$. 


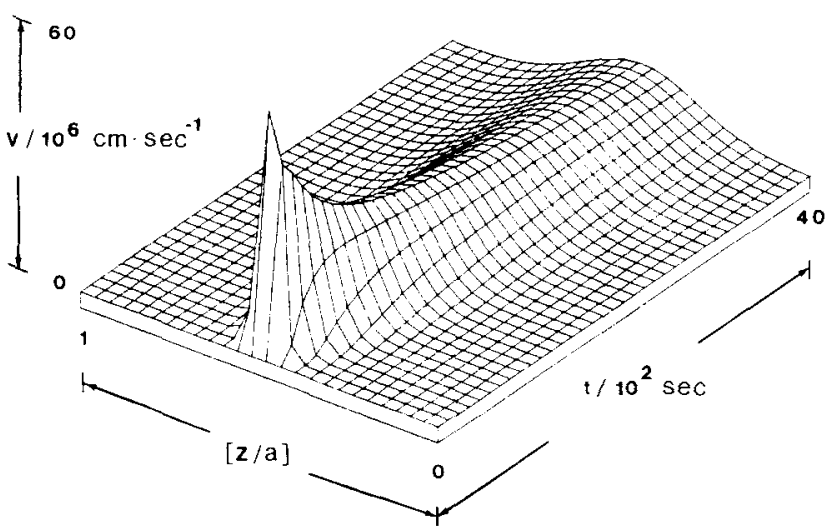

FIG. 2. Barycentric velocity surface for Run I.

\section{NUMERICAL SCHEME}

Equations (2.12), (2.16), and (2.10) were solved numerically with the Crank-Nicholson second-ordercorrect finite difference analogs for the time and spatial derivatives. ${ }^{8}$ The initial experimental conditions need not be isothermal to observe the diffusion thermoeffect as long as the initial temperature distribution (it must be small enough to avoid thermal diffusion terms) is known in order to assign values to the first row of grid points. The initial conditions used were

$$
\begin{aligned}
& x_{1}[0 \leq(z / a)<0.5]=x_{1}^{\mathrm{L}}, \quad x_{1}[0.5<(z / a) \leq 1]=x_{1}^{\mathrm{U}}, \\
& T[(z / a), 0]=T(z / a),
\end{aligned}
$$

where $x_{1}^{\mathrm{L}}$ and $x_{1}^{\mathrm{U}}$ are the initial $\mathrm{CCl}_{4}$ mole fractions in the lower and upper layers, respectively, and $T(z / a)$ is the measured temperature distribution at the moment of interface formation. The composition at $(z / a)=0.5$ is an arithmetic mean mole fraction but need not be defined unless a grid point in the numerical scheme is located at the interface.

The experimentally imposed boundary condition for the temperature equation,

$$
(\partial T / \partial z)_{0, t}=0=(\partial T / \partial z)_{a, t},
$$

corresponds to adiabatic cell walls and is incorporated into the numerical integration scheme via a reflective boundary condition. The same numerical procedure was used when imposing the boundary conditions of Eq. (2.14) on either Eq. (2.15) or (2.16).

Numerical stability was checked by comparison of results obtained using different step sizes. The number of spatial and temporal grid divisions was varied by more than an order of magnitude without change in results. Comparison of the numerical solution to $\mathbf{E q}$. (2.17) provided a further check on the numerical results.

In using the Crank-Nicholson method, an entire time row of grid points must be calculated simultaneously. Solution of the composition equation, Eq. (2.16), along the $n$th time row allowed inclusion of the composition dependence of the properties and parameters which appear in Eq. (2.10) before subsequent solution of the temperature equation along the same $n$th row. The temperature distribution used in the calculation of property values along the $n$th row was, however, that of the $n-1$ st row. Because temperature changes throughout the experiment were small, an iterative evaluation of the $n$th row temperature distribution was thereby avoided. Nevertheless, time step sizes were kept small to insure the accuracy of the above method.

A numerical solution of the temperature equation is shown in Fig. 3. Note that the upper or less dense phase rapidly increases in temperature after initial interface formation while the lower phase decreases. This conveniently eliminates density inversion possibilities and reflects a positive heat of transport $\bar{Q}_{1}^{*}$. The maximum $\Delta T$ between the top and bottom phases shown in Fig. 3 is about $0.28^{\circ} \mathrm{K}$. Rapid establishment of the maximum is due to the large initial composition gradient which then slowly decays as shown in Fig. 1.

In confirmation of the results described in DE. I. , numerical simulation shows that the heat of mixing contribution to the local temperature distribution is symmetric about the interface. While an endothermic heat of mixing lowers and an exothermic heat of mixing raises the overall temperature of the cell, the difference in temperature between two points symmetric about the interface is not affected by the heat of mixing term. Even mixtures in which $\bar{H}^{E}$ differs greatly from that of regular solution theory allow determination of $\Delta T$ between points symmetric about the interface without interference from the heat of mixing. Although heat of mixing data are fully included in the equations, only temperature differences measured at loci equidistant from the interface are used in the determination of the heat of transport. This method essentially subtracts out the relatively large background temperature change (with its accompanying uncertainties), so that smaller Dufour effect-related temperature differences can be measured.

Data analysis was accomplished by insertion of the numerical integration program into a generalized, weighted, nonlinear least-squares fitting routine known as "KINFIT4." "10 The heat of transport was obtained from the fit of predicted-to-measured $\Delta T$ values as a function of time. The properties involved in Eq. (2.10) for

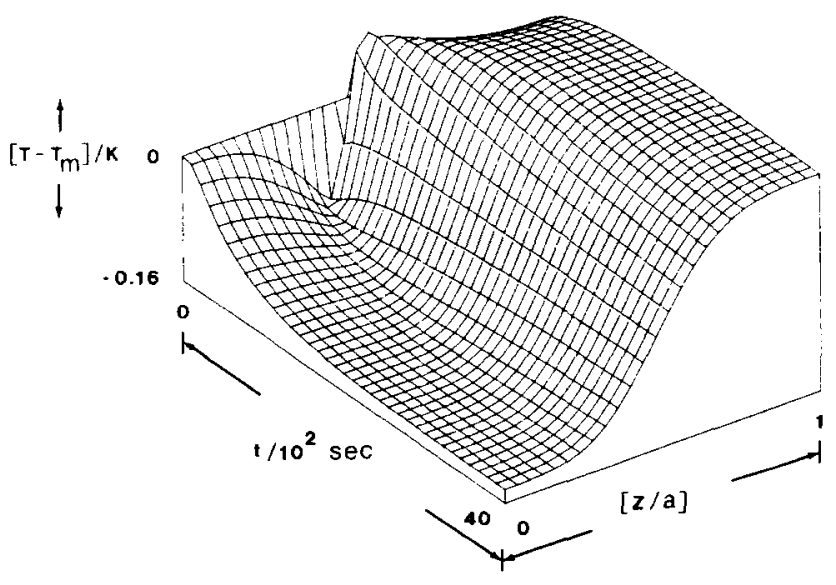

FIG. 3. Temperature surface for Run I. 
the carbon tetrachloride-cyclohexane system were obtained from the literature and appear in Table I. The original literature data have been put in the form

$$
\begin{aligned}
L & =L_{0}\left[1+L_{x}\left(x_{1}-0.5\right)+L_{r}(T-298.15)\right. \\
& \left.+L_{x T}\left(x_{1}-0.5\right)(T-298.15)+\frac{1}{2} L_{x x}\left(x_{1}-0.5\right)^{2}\right],
\end{aligned}
$$

where $L$ is the property in question, $L_{0}$ is the value of $L$ for an equimolar mixture at $298.15^{\circ} \mathrm{K}$, and $L_{x}, L_{T}$, $L_{x T}$, and $L_{x x}$ are composition and temperature derivatives. Some pure component values $L_{1}^{0}$ and $L_{2}^{0}$ are also listed.

Values for two properties are absent from Table I: the heat of transport, and the thermal conductivity of the mixture. Since the purpose of this research was the determination of $\tilde{Q}_{1}^{*}$, we were careful not to assume anything about its value or its composition dependence beforehand. In particular, we did not a priori assume the validity of the Onsager Reciprocal Relation.

The thermal conducitivty of the mixture must be known in order to determine the heat of transport. The only reported experiments for this mixture are by Venart. ${ }^{16,17}$ His results show a most peculiar cusp at $x_{1} \simeq 0.5$ when $\kappa$ is plotted against $x_{1}$. No other nonelectrolyte mixture displays this behavior. ${ }^{16,17}$ Rather than use Venart's data, we instead obtain the composition dependence of $\kappa$ from our Dufour effect experiments. We follow an iterative procedure in which we first neglect the composition dependence of the heat of transport and then include its experimental value in order to determine the composition dependence of the thermal conductivity. To illustrate the approach, consider the temperature equation without the very small term due to the barycentric velocity,

$$
\begin{gathered}
\tilde{C}_{p}(\partial T / \partial t)=\tilde{V}_{\kappa}\left(\partial^{2} T / \partial z^{2}\right)+\tilde{V}\left(\partial \kappa / \partial x_{1}\right)_{T, p}\left(\partial x_{1} / \partial z\right)(\partial T / \partial z) \\
+D\left(M_{1} \tilde{Q}_{1}^{*} / \tilde{M}\right)\left(\partial^{2} x_{1} / \partial z^{2}\right)+D\left(\partial^{2} \tilde{H}^{E} / \partial x_{1}^{2}\right)_{T, p}\left(\partial x_{1} / \partial z\right)^{2} \\
+D\left[\partial\left(M_{2} \tilde{Q}_{1}^{*} / \tilde{M}\right) / \partial x_{1}\right]_{T, p}\left(\partial x_{1} / \partial z\right)^{2} .
\end{gathered}
$$

In the first fitting of the experimental data, the last term on the right hand side of Eq. (3.4) was omitted. The calculations gave $\tilde{Q}_{1}^{*}$ and a first approximation for $\left(\partial \kappa / \partial x_{1}\right)_{T, P}$. [Values of the other parameters are known - see Table I.] Once $\tilde{Q}_{1}^{*}$ was obtained for all five experiments, its composition dependence was then included, and Eq. (3.4) was used, in full, to obtain an improved estimate of $\left(\partial \kappa / \partial x_{1}\right)_{T, P}$.

Although use of $\Delta T$ data rather than values for $T$ itself did not allow accurate simultaneous determination of two parameters for each run, inclusion of multiple run information, in the above described iterative fashion, decoupled the two parameters and allowed accurate determination of both the heat of transport and the thermal conductivity.

\section{EXPERIMENTAL}

"Baker Analyzed" spectrophotochemical reagent grade carbon tetrachloride and cyclohexane were used without further purification. Both reagents had lot analyses with guaranteed purities of $99.0 \%$.

\section{Interface formation}

For analysis of experimental data using the previously derived equations, an initially sharp diffusional interface must be instantaneously created between two layers of different $\mathrm{CCl}_{4}$ and $c-\mathrm{C}_{6} \mathrm{H}_{12}$ concentrations. At the moment of interface formation, the cell must be changed from an isothermal to an adiabatic configuration in accord with the mathematical initial boundary conditions. Traditional diffusion cells use mechanical methods of interface creation, often followed by siphon boundary sharpening. Although Dufour effect experiments require a distinct, sharp interface like that of diffusion experiments, the method of creation is more important since the response monitored is temperature rather than composition. Characteristic times are much shorter for thermal effects than for diffusion and boundary sharpening techniques are too slow to prevent heat conduction. Mechanical interfacial formation such as slide withdrawal or cell rotation can introduce turbulence as well as obscure the initial time of the experiment. The experimental starting time is uncertain due to finite times of slide withdrawal or cell rotation during which only part of the interface has been formed. Turbulence and initial time problems are coupled. If the mechanical motion is accelerated to reduce time errors, interfacial turbulence is enhanced. ${ }^{18}$

In order to achieve a sharp interface without turbulence, at a well-defined initial time, we designed a Dufour cell in which the sharp interface remains after the slow withdrawal of a third component from between the upper and lower layers. No ambiguity of the initial time is introduced since the third component is immiscible in the other two layers. Thus, diffusion is prevented until the middle layer has been completely withdrawn, whereupon the two layers of interest come into contact. Interfacial turbulence is minimized, and there are no seals or possible leakages in the interfacial region. Unfortunately, the binary systems amenable to investigation with this cell are restricted to those for which a third component can be found possessing the necessary properties: (1) a density intermediate to the densities of the upper and lower mixtures, and (2) insignificant solubility in either of the other two components. Distilled water satisfies these requirements for the carbon tetrachloride-cyclohexane system and therefore served as the withdrawable "liquid gate" for the experiments reported here.

The glass cell shown in Fig. 4 has two sections internally separated by an $8.5 \mathrm{~cm}$ length of glass tubing approximately $35 \mathrm{~mm}$ i.d. containing a stopcock. The upper and lower sections are jacketed for either thermostatting or vacuum insulating the containers. A stopcock in the tube connecting these jackets allows a vacuum to be created around only the lower cell. The bottom container is the diffusion thermoeffect cell. The upper container serves only as a reservoir for the less dense phase during displacement of the withdrawable liquid gate. The inside cell height of the diffusion thermoeffect chamber is $2.0 \mathrm{~cm}$.

The cell was filled in preparation for each experi- 


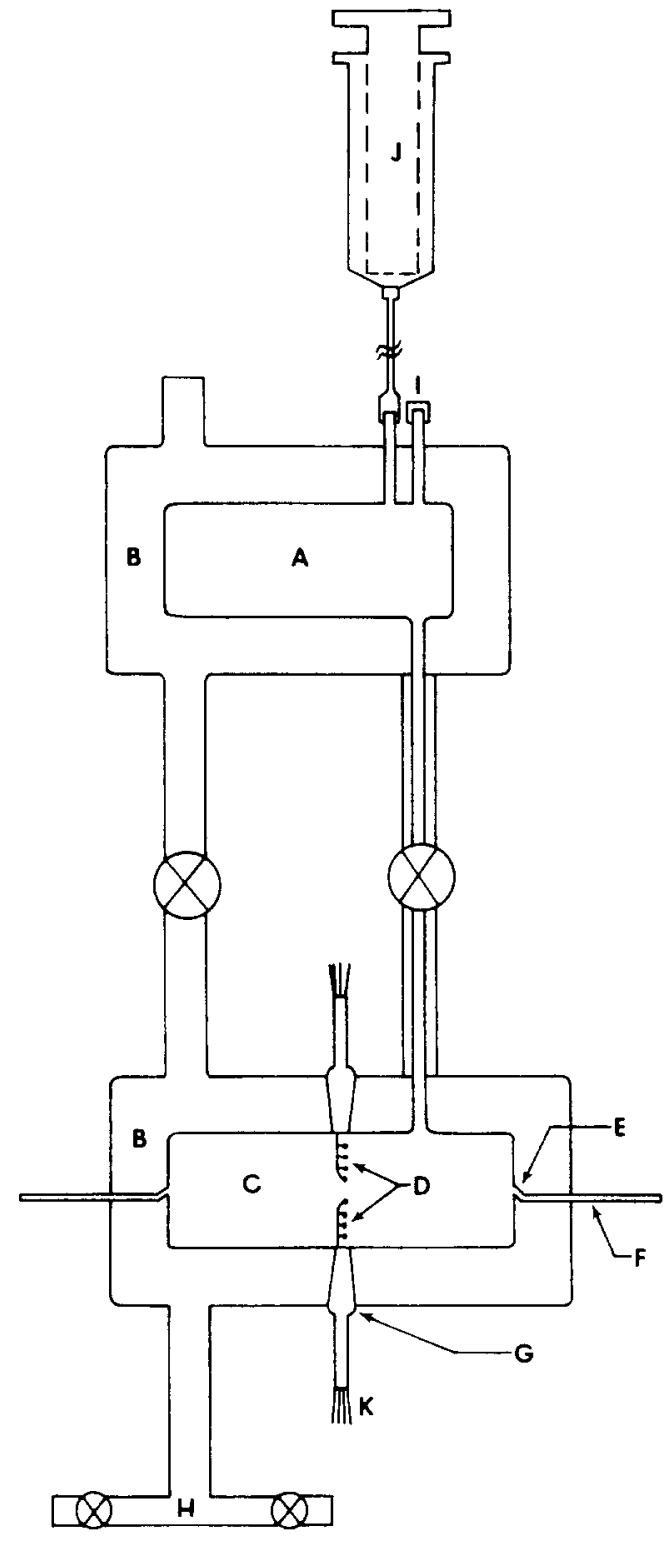

FIG. 4. Schematic diagram of withdrawable liquid gate diffusion thermoeffect cell. A. Upper phase storage reservoir. B. Cell jacket for thermostatting or adiabatically insulating. C. Diffusion thermoeffect chamber. D. Thermocouple banks. E. Equatorial water entrapment rim. F. "Liquid gate" withdrawal spout. G. Ground glass fittings for thermocouple leads and cell drainage. H. T connector to vacuum line and thermostat. I. Filling tubes. J. Glass syringe.

mental run by layering the carbon tetrachloride rich mixture beneath the distilled water layer. This prevented evaporational composition changes during cell infusion. The cyclohexane rich layer was then introduced into the top sto rage cell container by means of a large syringe. The syringe was left connected to the storage reservoir during withdrawal of the liquid gate. It served as an enclosed piston for volume displacement as the distilled water was withdrawn.

After filling, the cell and storage reservoir were thermally equilibrated with the thermostatting jacket surrounding them. The change from isothermal to adiabatic conditions was made by evacuating the jacket with a vacuum pump. It was found that adiabaticity could not be achieved if a liquid was circulated in the circumambient jacket as the thermostatting fluid. Wetting of the cell walls by a thermostatting liquid left residual droplets when the jacket was drained. Vaporization (and associated heat effects) of the droplets as the jacket was evacuated prevented the achievement of adiabaticity. Consequently, room temperature air served as the thermostatting fluid. No temperature effects were observed when the jacket was evacuated for a trial run in which the cell contained only pure water.

The liquid gate (distilled water) was withdrawn through four equatorial spouts by a.syringe pump at a rate of $0.764 \mathrm{~mL} / \mathrm{min}$ until only a small phase separated the two carbon tetrachloride-cyclohexane layers. From this point until contact of the two layers, withdrawal rates were slowed to $0.0206 \mathrm{~mL} / \mathrm{min}$ to prevent convection currents. Smooth interface creation occurred uniformly and isochronously throughout the cell except within the equatorial rim, shown in Fig. 4, where the meniscii were curved by preferential wetting. Due to this wetting, any residual water at the time of contact between the upper and lower layers was contained within the rim. Preliminary experiments indicated that constriction of interfacial diameter relative to cell bulk diameter reduced the ensuing temperature gradient. This is due to radial thermal conduction in regions of the cell above and below the residual water phase. Care was taken to ensure that any residual water at the time of interface formation was contained within the small bulge or rim from which the withdrawal spouts extended.

The experimental technique for interfacial formation is extremely important in Dufour experiments. The liquid gate method presented here has the obvious advantage of rapid and uniform interface formation without the agitation and leakage problems associated with typical diffusion cells which utilize moving parts for boundary formation. The initial interface formed after extraction of the liquid gate is very sharp and distinct. The absence of diffusion perturbing monolayers is assumed because of the hydrophobic nature of both carbon tetrachloride and cyclohexane. Furthermore, monolayers, if initially formed, cannot persist for long because the system soon becomes continuous as diffusion proceeds to level the initial step function of composition. Nevertheless, a slight shifting of the diffusional time scale may occur if monolayers are initially present.

\section{Temperature measurement and data analysis}

Immediately upon interface formation, a Precision Scientific Co "time it" digital timer ( $0.1 \mathrm{sec}$ readout) was activated, the syringe pump was disengaged, the stopcock between the reservoir and the cell was closed, and the vacuum jacket was evacuated. Temperature changes were monitored with copper-constantan thermocouples located symmetrically about the interface. Ice bath referenced potentials were read from a Leeds and Northrup Co. $K-3$ potentionmeter facility which provided $\pm 0.002{ }^{\circ} \mathrm{K}$ precision. Exact thermocouple locations were measured in situ with a Beck Vernier Measuring Microscope. Accuracy in thermocouple locations was 
TABLE II. Heat of transport for carbon tetrachloride (1)cyclohexane (2) mixtures.

\begin{tabular}{|c|c|c|c|c|c|c|}
\hline \multirow[b]{2}{*}{ Run } & \multicolumn{5}{|c|}{ Initial conditions } & \multirow{2}{*}{$\begin{array}{l}\text { Heat of } \\
\text { Transport } \\
\tilde{Q}_{1}^{*} / \mathrm{kJ} \mathrm{mol}^{-1}\end{array}$} \\
\hline & $x_{1}^{U}$ & $x_{1}^{L}$ & $\left\langle x_{1}\right\rangle$ & $\Delta x_{1}$ & $T /{ }^{\circ} \mathrm{K}$ & \\
\hline I & 0.0179 & 0.8044 & 0.3469 & 0.5933 & 296.160 & 5.47 \\
\hline II & 0.0503 & 0.6436 & 0.4112 & 0.7865 & 295.355 & 5. 59 \\
\hline III & 0.0951 & 0.7638 & 0.4295 & 0.6687 & 295. 131 & 5.43 \\
\hline $\mathrm{N}$ & 0.0750 & 0.8935 & 0.4843 & 0.8185 & 296.020 & 5. 84 \\
\hline v & 0.1934 & 0. 9094 & 0.5514 & 0.7160 & 296.429 & 6. 13 \\
\hline
\end{tabular}

${ }^{\mathrm{a}}$ Maximum root mean-square deviation between experimental and fitted data for each run was $0.12 \mathrm{~kJ} \mathrm{~mol}^{-1}$.

limited by the finite size of the welded thermocouple junctions (usually $0.2 \mathrm{~mm}$ in diameter).

The maximum temperature difference between symmetric thermocouples was obtained after 500-800 sec. The maximum temperature difference depends upon the difference in initial compositions, in accordance with the results of DE. I. The experimental starting mole fraction differences varied between 0.59 and 0.82 , with corresponding temperature difference maxima from $0.21^{\circ} \mathrm{K}$ to $0.30^{\circ} \mathrm{K}$, respectively. Because preliminary experiments indicated that some turbulence was caused upon contact of the two layers if pure components rather than mixtures were used, only mixtures were used for the initial phases. The five different sets of initial conditions are listed in Table II.

Because the boundary conditions used for solving the temperature equation were for adiabatic walls, only the thermocouple pair closest to the interface was used in analysis of the data. The innermost pair is expected to be least susceptible to errors resulting from: (1) perturbations due to the presence and finite size of the thermocouple holder and the other thermocouples, (2) thermal conduction through the thermocouple holders, (3) side wall effects, and (4) heat loses through the cell ends; i. e., nonconformity to the prescribed boundary conditions. The first problem was minimized by allowing the thermocouple junctions to protrude $1.5 \mathrm{~mm}$ from the thermocouple holder. In addition, the innermost thermocouple of each group extended $1.0 \mathrm{~mm}$ below the holders to reduce the second possible source of error. The large diameter-to-height ratio makes any side wall effects negligible in the region of the temperature sensors for the pair closest to the interface. Figure 5 is a comparison of the time behavior for $\Delta T$ measured between the positions $(z / a)=0.4$ and $(z / a)=0.6$ and $\Delta T$ obtained by computer simulation for (1) adiabatic boundary conditions and (2) diathermic boundary conditions. The boundary conditions change the time dependent behavior of the calculated $\Delta T$ values considerably. The actual $\Delta T$ time dependence corresponds to that predicted on the basis of adiabatic walls. Thermocouple pairs located further from the interface also agree with calculated $\Delta T$-time shapes for the adiabatic model at shorter times but began to deviate at longer times. Figure 5 shows that the innermost thermocouple pair provides temperature difference data in accord with the assumed adiabatic boundary conditions for the experimental time scale ( $4000 \mathrm{sec})$. A comparison run in which the innermost thermocouples were each moved $10 \%$ further from the interface yielded the same value for the heat of transport.

From Fig. 5, if only the first $600 \mathrm{sec}$ of the experiment were analyzed, the value obtained for $\tilde{Q}_{1}^{*}$ would be slightly lower than it is for the time interval between 1200 and $3000 \mathrm{sec}(\Delta T$ is essentially proportioned to $\bar{Q}_{1}^{*}$ ). Stability of the parameter estimate for $\tilde{Q}_{1}^{*}$ was checked as a function of the time range over which data points were input. This data truncation test showed that the parameter estimate remains unchanged within $1 \%$ for inclusion of data past $2800 \mathrm{sec}$ and remains virtually unchanged when data past $3400 \mathrm{sec}$ are included.

Two further effects are noteworthy: (1) Since data were taken at roughly $100 \mathrm{sec}$ intervals, inclusion of longer time data requires inclusion of more data points, which leads to better statistics. (2) The parameter being fit, $\mathrm{A} \equiv\left(\bar{Q}_{1}^{*} / \bar{M}\right)$, is not independent of composition which leads to less accuracy in the fitting procedure at short times. Inclusion of data taken at relatively longer times is important in order to find A for each run's mean composition because at short times two very different compositions are located on either side of the interface. As diffusion levels the initially sharp composition gradient in the interfacial region, the assumed model ( $\mathrm{A}$ is constant) becomes more nearly correct. Analysis of the experiments reported here included data taken up to $4200 \mathrm{sec}$.

The custom in gaseous Dufour effect experiments ${ }^{5}$ of using only the maximum $\Delta T$ to calculate the heat of transport is inaccurate, compared to our technique, in which $\Delta T$ vs time data are used. Integration of the appropriate partial differential equations and fitting the time dependent behavior of the measured temperature differences provides a statistically better estimate of the heat of transport, particularly since the maximum temperature difference occurs at relatively short times where poor interface formation can affect the results.

Although $\Delta T$ data were used in data reduction, absolute temperatures were also measured. Unlike the $\Delta T$ vs time curves, measured $T$ vs time profiles were quite

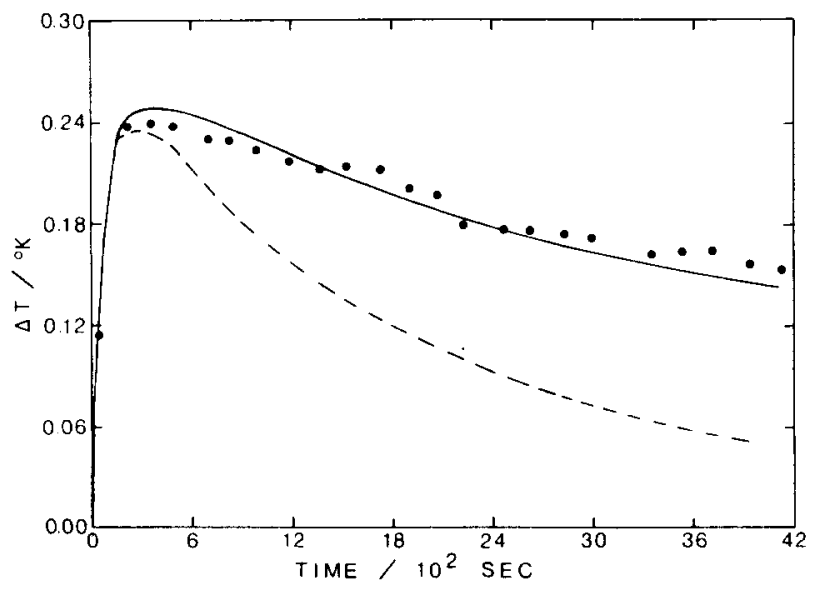

FIG. 5. Comparison of experimental data for Run I (dots) to calculated values for adiabttic walls (__ ) and for diathe rmic walls $(--)$. 
asymmetric about $(z / a)=0.5$. In all cases, the increase from the uniform initial temperature was much less for the thermocouple located above the interface than was the decrease in temperature for the corresponding lower thermocouple of the pair. This asymmetric effect is analogous to that observed by Mason, Miller, and Spurling, ${ }^{5}$ by Waldmann, ${ }^{4}$ and by Miller ${ }^{19}$ in their gaseous Dufour effect experiments. These investigators all noted that the temperature effect was greater below the diffusion interface than above it. Our computer simulation concurs with the hypothesis for this effect advanced by Mason et al. ${ }^{5}$ - the asymmetry in the temperature effect is primarily due to composition dependencies of the transport parameters, particularly the thermal conductivity. Individual thermocouples were not used to fit the composition dependence of the thermal conductivity, however, because of the large background contribution from the heat of mixing. Theoretical $T$ vs $t$ plots for a given cell position agreed with observed behaviors at shoríer times. Deviations at intermediate and longer times were observed, however. Reasons for this are not known but may be due to inaccuracies in the second derivative of the excess enthalpy as required by Eq. (2.10).

\section{RESULTS}

For each of the five runs the $\Delta T$ vs time data were first fit to the numerical solution of

$$
\begin{aligned}
\tilde{C}_{p}(\partial T / \partial t)= & \tilde{V} \kappa_{0}\left(\partial^{2} T / \partial z^{2}\right)+\tilde{V}\left(\partial \kappa / \partial x_{1}\right)_{T, P}\left(\partial x_{1} / \partial z\right)(\partial T / \partial z) \\
& +D M_{2} A\left(\partial^{2} x_{1} / \partial z^{2}\right)+D H_{x x}^{E}\left(\partial x_{1} \partial z\right)^{2}
\end{aligned}
$$

which is obtained from Eq. (3.4) by omitting the last term of Eq. (3.4). In computations using Eq. (5.1), all coefficients were evaluated as functions of composition from the data of Table I, with $H_{x x}^{E} \equiv\left(\partial^{2} H^{E} / \partial x_{1}^{2}\right)_{T, P}$, and with $A \equiv\left(\tilde{Q}_{1}^{*} / \tilde{M}\right)$ taken to be constant. A weight fraction average of pure component thermal conductivities was used to make a first estimate of the thermal conductivity, $\kappa_{0}$. Thus, in the first computations, both $\left(\partial_{K}\right)$ $\left.\partial x_{1}\right)_{T, P}$ and $A$ are obtained. Once $A$ has been obtained for different mean compositions, then the last term of Eq. (3.4) is reinstated, and the data are reanalyzed to obtain new values of $\left(\partial \kappa / \partial x_{1}\right)_{T, P}$ and $A$ by means of

$$
\begin{aligned}
\tilde{C}_{b}(\partial T / \partial t)= & \kappa \tilde{V}\left(\partial^{2} T / \partial z^{2}\right)+\tilde{V}\left(\partial \kappa / \partial x_{1}\right)_{T, p}\left(\partial x_{1} / \partial z\right)(\partial T / \partial z) \\
& +D M_{2} A\left(\partial^{2} x_{1} / \partial z^{2}\right)+D H_{x x}^{E}\left(\partial x_{1} / \partial z\right)^{2} \\
& +D M_{2}\left(\partial A / \partial x_{1}\right)_{T, p}\left(\partial x_{1} / \partial z\right)^{2},
\end{aligned}
$$

where $\kappa$ is obtained from pure component thermal conductivities and the previously determined $\left(\partial \kappa / \partial x_{1}\right)_{T, P}$.

\section{Heat of transport}

The results for $\tilde{Q}_{1}^{*}=\tilde{M} A$ are listed in Table $\Pi$. A linear least-squares fit of the data yields

$\tilde{Q}_{1}^{*}\left(\mathrm{~kJ} \mathrm{~mol}^{-1}\right)$

$$
=5.82+2.32\left(x_{1}-\frac{1}{2}\right)+0.225(T-295.82) \text {. }
$$

Values of $\tilde{Q}_{1}^{*}$ calculated from Eq. (5.3) match the observed values with a root mean-square deviation of 0.087 , about $1.5 \%$. Note that $\tilde{Q}_{1}^{*}$ is strongly composition dependent, with $\bar{Q}_{1}^{*-1}\left(\partial \bar{Q}_{1}^{*} / \partial x_{1}\right)_{T, P}=0.399$ for $x_{1}=\frac{1}{2}$. The mean molecular weight is also strongly composition dependent, with $\tilde{M}^{-1}\left(d \tilde{M} / d x_{1}\right)=0.585$ for $x_{1}=\frac{1}{2}$. The ratio $A=\left(\tilde{Q}_{1}^{*} / \tilde{M}\right)$ is less composition dependent, with $A^{-1}\left(\partial A / \partial x_{1}\right)_{T, P}=-0.186$ for $x_{1}=\frac{1}{2}$.

We have previously reported ${ }^{3}$ the successful testing of the Onsager Reciprocal Relation $\Omega_{01}=\Omega_{10}$ by comparing the results of Eqs. (5.3) and (2.7) with the results of thermal diffusion experiments. We have also shown ${ }^{3}$ that thermal diffusion factors calculated from heats of transport according to

$$
\alpha_{1}=-\tilde{Q}_{1}^{*} M_{1} M_{2}\left[\tilde{M}\left(\partial \tilde{\mu}_{1} / \partial \ln x_{1}\right)_{T, P}\right]^{-1}
$$

agree with results of thermal diffusion experiments well within the mutual experimental uncertainties.

\section{Thermal conductivity}

In obtaining $\left(\partial \kappa / \partial x_{1}\right)_{T, P}$, we use the NEL equation ${ }^{16,20}$

$$
\kappa=w_{1} \kappa_{1}^{0}+w_{2} \kappa_{2}^{0}-C\left(\kappa_{2}^{0}-\kappa_{1}^{0}\right)\left(1-w_{2}^{1 / 2}\right) w_{2},
$$

where $w_{i}$ is mass fraction,

$$
w_{i}=x_{i} M_{i} \tilde{M}^{-1} \text {, }
$$

$\kappa_{i}^{0}$ is the thermal conductivity of pure component $i$, and $C$ is a constant. Jamieson and co-workers have shown ${ }^{16,20}$ that most nonelectrolyte mixture thermal conductivities are fit best by Eq. (5.5) when $C=1.0$. From Eqs. (5.5) and (5.6),

$$
\left(\partial \kappa / \partial x_{1}\right)_{T, P}=M_{1} M_{2} \bar{M}^{-2}\left(\kappa_{1}^{0}-\kappa_{2}^{0}\right)\left[1-C\left(1-\frac{3}{2} w_{2}^{1 / 2}\right)\right] \text {. (5. 7) }
$$

The first computation showed that the data were best fit by Eq. (5.1) when $C=0.6$. The second computation showed that the data were best fit by Eq. (5.2) when the values of $\bar{Q}_{1}^{*}$ from Table II were used and when $C=1.05$. Thus, the composition dependence of the thermal conductivity of the carbon tetrachloride-cyclohexane mixtures studied in the work reported here is best given by

$$
\left(\partial_{\kappa} / \partial w_{1}\right)_{T_{,} P}\left(\mathrm{~J} \mathrm{~m}^{-1} \mathrm{~s}^{-1} \mathrm{k}^{-1}\right)=0.0252\left(w_{2}^{1 / 2}-0.032\right),(5.8)
$$

where we have used the pure component values of Table I.

\section{DISCUSSION}

The Dufour effect in liquid mixtures has long been assumed to be too small or too complicated for accurate experimental determination of heats of transport. We have used a liquid gate withdrawal cell with adiabatic cell walls to observe the Dufour effect quantitatively. Nonlinear, weighted, least-squares analysis of the measured temperature differences between thermocouples positioned equidistant from the interface yields accurate values for the heat of transport in carbon tetrachloridecyclohexane mixtures. Onsager coefficients obtained from the measured heats of transport provided ${ }^{3}$ the first experimental verification of the Onsager heat-mass and mass-heat reciprocal relation when compared to Onsager coefficients obtained from literature thermal diffusion data.

For carbon tetrachloride-cyclohexane mixtures, the composition dependences of both the thermal conductivity and the heat of transport, as well as the heat of transport itself, were taken as a priori unknown parameters in the numerical fitting of the data. Although our re- 
sults indicate that the thermal conductivity of carbon tetrachloride-cyclohexane mixtures obeys the NEL equation $^{16,20}$ for intermediate compositions, considerable experimental and numerical refinement would be necessary before the Dufour effect could be used to determine mixture thermal conductivities routinely. The iterative numerical procedure used in this work decouples the calculation of the heat of transport from the mixture thermal conductivity and yields accurate values of $\tilde{Q}_{1}^{*}$.

The molecular theory of the Dufour effect in nonequilibrium liquid mixtures has seen little progress since the work of Bearman, Kirkwood, and Fixman. ${ }^{21}$ Our recent ${ }^{22}$ experimental determination of the temperature dependence of $\bar{Q}_{1}^{*}$ for nearly critical mixtures of isobutyric acid and water suggests ${ }^{22}$ that the heat of transport is, as expected, closely coupled to mutual diffusivity on the molecular scale. Since five very different kinds of experiments all give the same numerical results for the thermal-diffusion and diffusion-thermal properties of carbon tetrachloride-cyclohexane liquid mixtures, ${ }^{3}$ this system can provide the reliable data required to test molecular theories of nonisothermal transport in mixtures far away from the critical region.

\section{ACKNOWLEDGMENT}

Support of RLR in 1978 through a General Electric Foundation-Michigan State University Summer Fellowship is gratefully acknowledged.

${ }^{1}$ F. H. Horne and R. J. Bearman, J. Chem. Phys. 37, 2842 (1962); J. L. Bartelt and F. H. Horne, ibid. 51, 210 (1969). ${ }^{2}$ L. Onsager, Phys. Rev, 37, 405 (1931); 38, 2265 (1931).

${ }^{3}$ R. L. Rowley and F. H. Horne, J. Chem. Phys. 68, 325 (1978).

${ }^{4}$ L. Waldmann, Z. Phys. 121, 501 (1943); 124, 2 (1947); 124, 30 (1947); 124, 175 (1948); Z. Naturforsch. A 1, 59 (1946);
2, 358 (1947); 4, 105 (1949).

${ }^{5}$ R. P. Rastogi and G. L. Madan, Trans. Faraday Soc. 62, 3325 (1966); E. A. Mason, L. Miller, and T. H. Spurling, J. Chem. Phys. 47, 1669 (1967):, B. L. Sawford, T. H. Spurling, and D. S. Thurley, Aust. J. Chem. 23, 1311 (1970); A. Bousheri, J. Chem. Eng. Data 19, 3136 (1974); A. Bousheri and S. Afrashtehfar, Bull. Chem. Soc. Jpn. 48, 2372 (1975).

${ }^{6}$ R. P. Rastogi and G. L. Madan, J. Chem. Phys. 43, 4179 (1965); R. P. Rastogi and B. L. S. Yadava, ibid. 51, 2826 (1969); 52, 2791 (1970).

${ }^{7}$ S. E. Ingle and F. H. Horne, J. Chem. Phys. 59, 5882 (1973).

${ }^{8}$ R. L. Rowley, Ph. D. Dissertation, Michigan State University, 1978.

${ }^{9}$ D. U. Rosenberg, Methods for the Numerical Solution of Partial Differential Equations (American Elsevier, New York, 1969).

10، KINFIT4," the 1977 version of the original "KINFIT" of J. L. Dye and V. A. Nicley, J. Chem. Educ. 48, 443 (1971), may be obtained from F. H. Horne.

${ }^{11} \mathrm{R}$. C. Weasts, editor, Handbook of Chemistry and Physics, 51st Ed. (The Chemical Rubber Co., Ohio, 1970).

${ }^{12}$ T. G. Anderson and F. H. Horne, J. Chem. Phys. 53, 2332 (1970).

${ }^{13}$ M. B. Ewing and K. N. Marsh, J. Chem. Thermodyn. 2, $351(1970)$.

${ }^{14} \mathrm{E}$. Wilhelm, M. Zettler, and H. Sackmann, Ber. Bunsenges. Phys. Chem. 78, 795 (1974); J. D. E. Grolier, E. Wilhelm, and M. H. Hamedi, ibid. 82, 1282 (1978).

${ }^{15} \mathrm{~S}$. E. Wood and J. A. Gray, III, J. Am. Chem. Soc. 74, 3729 (1962).

${ }^{16}$ D. T. Jamieson, J. B. Irving, and J. S. Tudhope, editors, Liquid Thermal Conductivity, a data survey to 1973 (H. M. S., Edinburgh, 1975).

${ }^{17} \mathrm{~J}$. E. S. Venart, Thermophysical Properties, 4th Symp. (Univ. Delaware, Delaware, 1968).

${ }^{18}$ O. Bryngdahl, Acta Chem. Scand. 12, 648 (1958).

${ }^{19}$ I. Miller, Z. Naturforsch. Teil A 4, 262 (1949).

${ }^{20} \mathrm{D}$. T. Jamieson and E. H. Hastings, Thermal Conductivity, Proc. 8th Conf., C. Y. Ho and R. E. Taylor, editors (Plenum, New York, 1969).

${ }^{21}$ R. J. Bearman, J. G. Kirkwood, and M. Fixman, Adv. Chem. Phys. 1, 1 (1958).

${ }^{22}$ R. L. Rowley and F. H. Horne, J. Chem. Phys. 71, 3841 (1979). 